\title{
Chronic kidney disease-induced atrial structural remodeling and atrial fibrillation: more studies on the pathological mechanism are encouraged
}

\author{
Huiliang Qiu ${ }^{1,2}$ (D) Chunlan $\mathrm{Ji}^{1,3} \cdot$ Huanlin $\mathrm{Wu}^{1,2,4} \cdot$ Chuan Zou ${ }^{1,3}$ \\ Received: 17 February 2018 / Accepted: 5 April 2018 / Published online: 10 May 2018 \\ (C) Springer-Verlag GmbH Germany, part of Springer Nature 2018
}

Dear Editor,

It is well known that chronic kidney diseases (CKD) increase the prevalence of atrial fibrillation (AF). Epidemiologic studies suggest CKD induce two- to threefold likelihood of AF, especially the end-stage renal disease (Soliman et al., 2010; Liao et al., 2015), yet the underlying mechanisms of AF pathogenesis in the case of CKD still remain unclear. As CKD is always accompanied with other risk factors of AF, such as hypertension, electrolyte disturbance, heart failure, and atrial enlargement, and complex pathological factors, such as inflammation, renin-angiotensin-aldosterone system (RAAS) activation and autonomic nervous system dysfunction, and the occurrence and development of atrial fibrillation, are considered multifactorial (McManus et al., 2012).

RAAS, transforming growth factor $\beta 1$ (TGF- $\beta 1$ ), and oxidative stress play important roles in atrial structural remodeling and in the development of AF (Everett 4th \& Olgin, 2007). RAAS can induce the activation of TGF- $\beta 1 /$ Smads pathway, and the latter can further induce ROS and oxidative stress in various disease situations (Everett 4th \& Olgin, 2007). Recent studies emphasize the important role of oxidative stress in the

Huiliang Qiu and Chunlan Ji contributed equally to this work.

Huiliang Qiu

vqiuhuiliang4837@163.com

$\triangle$ Chuan Zou

doctorzc541888@126.com

1 Second Clinical Medical College, Guangzhou University of Chinese Medicine, Guangzhou 510405, China

2 Department of Cardiology, Guangdong Provincial Hospital of Chinese Medicine, Guangzhou 510120, China

3 Department of Nephrology, Guangdong Provincial Hospital of Chinese Medicine, Guangzhou 510120, China

4 Beijing University of Chinese Medicine, Beijing 100700, China genesis of AF in the setting of CKD. A study by Fukunaga et al. successfully investigated the role of oxidative stress which mediated by nicotinamide adenine dinucleotide phosphate (NADPH) oxidase in the genesis of in the setting of 4 weeks post-5/6 nephrectomy rats, and demonstrated the antioxidant agent, sodium zinc dihydrolipoylhistidinate, is effective in reducing atrial oxidative stress, fibrosis, and AF inducibility (Fukunaga et al., 2012). In the similar animal setting, Aoki et al. reported that renal dysfunction-induced uremic toxin indoxyl sulfate is also associated with increased oxidative stress, inflammation, profibrotic factors, and AF (Aoki et al., 2015). However, the role of TGF- $\beta 1 /$ Smads pathway in $\mathrm{CKD}$-induced AF is still not fully understood, and further research is needed.

Though it is likely that oxidative stress mediated by NADPH oxidase plays a role in AF genesis, it is still inadequate to explain the whole pathological mechanism of $\mathrm{AF}$ in CKD. NADPH oxidase activity which is regulated by Rac1 is critical to the generation of oxidative stress (Adam et al., 2010; Satoh et al., 2006). Previous studies indicated that Ang II increases the protein expression of connective tissue growth factor (CTGF) via the activation of Rac1, resulting in the abnormal gap junction remodeling evidenced by the upregulation of $\mathrm{N}$-cadherin and connexin43 ( $\mathrm{Cx} 43)$ (Adam et al., 2010). However, it is unknown whether gap junction would be affected by CKD. Since gap junction remodeling is highly associated with $\mathrm{AF}$, any alterations in atrial $\mathrm{Cx} 43$ expression, phosphorylation, and distribution are considered proarrhythmogenic by affecting cell-to-cell electrical coupling, which will help solve a part of puzzles pointing to $\mathrm{CKD}$-induced atrial remodeling and $\mathrm{AF}$.

Calcium-handling abnormalities are known to play an important role in the pathophysiology of AF. Many pathological states are contributed to atrial $\mathrm{Ca} 2+$ overload and spontaneous $\mathrm{Ca} 2+$ leakage, leading to the delayed after depolarizations, triggered activity, re-entry, and atrial remodeling (Heijman et al., 2015). Recent studies reported that calcium-handling 
abnormalities are involved in uremic toxin indoxyl sulfate (IS)-induced AF. Chen et al. showed that treatment with IS increased pulmonary vein (PV) delayed after depolarizations and burst firings, reduced the sinoatrial node (SAN) spontaneous beating rate, and shortened the action potentials (AP) of isolated rabbit left atrium (LA). Similarly, after treatment with IS, the PV cardiomyocytes had a larger calcium transient, sarcoplasmic reticulum calcium content, and calcium leak. However, using antioxidant ascorbic acid attenuated the effects of IS on the LA, PV, and SANs (Chen et al., 2015). Besides, Huang et al. demonstrated that rabbits with CKD, induced by intraperitoneal injection of a mixture of neomycin sulfate and cefazolin for 4 weeks, developed PV arrhythmogenesis with an enhanced calcium-handling abnormalities - a larger calcium transient amplitudes, calcium contents in sarcoplasmic reticulum, and a denser current of sodium/calcium exchanger and late sodium currents, but smaller L-type calcium current densities were found in PV cardiomyocytes - via protein kinase A and ROS (Huang et al., 2017). Indeed, oxidative stress can induce calciumhandling abnormalities, leading to the pathogenesis of AF. However, there are no specific studies investigating the role of calcium-handling abnormalities in CKD-induced $\mathrm{AF}$ in a point of mechanism to date.

What is more, recent studies indicated that the nucleotidebinding domain leucine-rich repeat-containing receptor (NLR) pyrin domain-containing protein 3 (NLRP3) inflammasome also plays a role in the genesis of atrial fibrosis (Li et al., 2017), and CKD participates in organ NLRP3 activation (Gong et al., 2016; Chin et al., 2017). NLRP3 inflammasome, a multiprotein complex, mediates the maturation of caspase-1, interleukin (IL)-1 $\beta$, and IL-18, boosting pathological inflammation. Previous studies have found an increased NLRP3 inflammasome activity in atrial tissue of both clinical AF patients and a mice model of spontaneous AF induced by CREM-Ib $\Delta \mathrm{C}-\mathrm{X} \mathrm{Tg}$ mice (Li et al., 2017; He et al., 2016). Further studies have demonstrated that the specific expression of NLRP3 develops premature atrial contractions and severe AF by constituting a CM-specific knock-in murine model which can express a constitutive active NLRP3. However, NLRP3 inhibition by using a selective inflammasome inhibitor significantly prevented AF ( $\mathrm{Li}$ et al., 2017). Besides, emerging evidences indicated that the NLRP3 inflammasome is involved in CKD-induced kidney fibrosis, whereas NLRP3 deletion inhibits renal fibrosis in the 5/6 nephrectomy (5/6 Nx) disease model (Gong et al., 2016). Furthermore, CKD induces the activation of NLRP3 not only in kidney but also in extrarenal organ. Chin et al. demonstrated that CKD-induced uremic toxin, the IS, is associated with the upregulation of NLRP3 inflammasome in ventricle and results in ventricular dysfunction (Chin et al., 2017). However, in the disease setting of CKD, the status of NLRP3 inflammasome/ caspase-1/IL-1 $\beta$ and IL-18 axis in atria is unknown.
Therefore, some questions are raised: (1) does TGF- $\beta 1 /$ Smads signaling involve in CKD-induced atrial fibrosis; (2) whether gap junction would be affected by $\mathrm{CKD}$, and how does the Cx43 change; (3) would atrial NLRP3 inflammasome/caspase-1/IL- $\beta 1$ and IL1 8 axis also be activated by $\mathrm{CKD}$; (4) are calcium-handling abnormalities targets for the prevention of $\mathrm{AF}$ in CKD? We have reasons to believe that the characteristics of CKD-induced atrial structural remodeling and $\mathrm{AF}$ are systemic and multifactorial. Further studies would be encouraged to investigate these pathological changes of CKD-induced AF.

\section{References}

Adam O, Lavall D, Theobald K, Hohl M, Grube M, Ameling S, Sussman MA, Rosenkranz S, Kroemer HK, Schäfers HJ, Böhm M, Laufs U (2010) Rac1-induced connective tissue growth factor regulates connexin 43 and $\mathrm{N}$-cadherin expression in atrial fibrillation. J Am Coll Cardiol 55(5):469-480

Aoki K, Teshima Y, Kondo H, Saito S, Fukui A, Fukunaga N, Nawata T, Shimada T, Takahashi N, Shibata H (2015) Role of indoxyl sulfate as a predisposing factor for atrial fibrillation in renal dysfunction. $\mathrm{J}$ Am Heart Assoc 4(10): 002023

Chen WT, Chen YC, Hsieh MH, Huang SY, Kao YH, Chen YA, Lin YK, Chen SA, Chen YJ (2015) The uremic toxin indoxyl sulfate increases pulmonary vein and atrial arrhythmogenesis. J Cardiovasc Electrophysiol 26(2):203-210

Chin LH, Hsu YJ, Hsu SC, Chen YH, Chang YL, Huang SM, Tsai CS, Lin CY (2017) The regulation of NLRP3 inflammasome expression during the development of cardiac contractile dysfunction in chronic kidney disease. Oncotarget 8(69):113303-113317

Everett TH 4th, Olgin JE (2007) Atrial fibrosis and the mechanisms of atrial fibrillation. Heart Rhythm 4(3 Suppl):S24-S27

Fukunaga N, Takahashi N, Hagiwara S, Kume O, Fukui A, Teshima Y, Shinohara T, Nawata T, Hara M, Noguchi T, Saikawa T (2012) Establishment of a model of atrial fibrillation associated with chronic kidney disease in rats and the role of oxidative stress. Heart Rhythm 9(12):2023-2031

Gong W, Mao S, Yu J, Song J, Jia Z, Huang S, Zhang A (2016) NLRP3 deletion protects against renal fibrosis and attenuates mitochondrial abnormality in mouse with 5/6 nephrectomy. Am J Physiol Renal Physiol 310(10):F1081-F1088

He G, Tan W, Wang B, Chen J, Li G, Zhu S, Xie J, Xu B (2016) Increased M1 macrophages infiltration is associated with thrombogenesis in rheumatic mitral stenosis patients with atrial fibrillation. PLoS One 11(3): e0149910

Heijman J, Voigt N, Ghezelbash S, Schirmer I, Dobrev D (2015) Calcium handling abnormalities as a target for atrial fibrillation therapeutics: how close to clinical implementation? J Cardiovasc Pharmacol 66(6):515-522

Huang SY, Chen YC, Kao YH, Hsieh MH, Lin YK, Chen SA, Chen YJ (2017) Redox and activation of protein kinase A dysregulates calcium homeostasis in pulmonary vein cardiomyocytes of chronic kidney disease. J Am Heart Assoc 6(7):e005701

Li N, Scott L, Yao C, Wehrens X, Dobrev D (2017) Activation of NLRP3 inflammasome plays a role in the pathogenesis of atrial fibrillation. Heart Rhythm 14(5, May Supplement) [Abstract]

Liao JN, Chao TF, Liu CJ, Wang KL, Chen SJ, Lin YJ, Chang SL, Lo LW, Hu YF, Tuan TC, Chung FP, Chen TJ, Chen SA (2015) Incidence and risk factors for new-onset atrial fibrillation among 
patients with end-stage renal disease undergoing renal replacement therapy. Kidney Int 87(6):1209-1215

McManus DD, Saczynski JS, Ward JA, Jaggi K, Bourrell P, Darling C, Goldberg RJ (2012) The relationship between atrial fibrillation and chronic kidney disease: epidemiologic and pathophysiologic considerations for a dual epidemic. J Atr Fibrillation 5(1):442

Satoh M, Ogita H, Takeshita K, Mukai Y, Kwiatkowski DJ, Liao JK (2006) Requirement of Rac1 in the development of cardiac hypertrophy. Proc Natl Acad Sci U S A 103(19):7432-7437
Soliman EZ, Prineas RJ, Go AS, Xie D, Lash JP, Rahman M, Ojo A, Teal VL, Jensvold NG, Robinson NL, Dries DL, Bazzano L, Mohler ER, Wright JT, Feldman HI, Chronic Renal Insufficiency Cohort (CRIC) Study Group (2010) Chronic kidney disease and prevalent atrial fibrillation: the Chronic Renal Insufficiency Cohort (CRIC). Am Heart J 159:1102-1107 